$17^{\text {th }}$ International Congress of Metrology, 01002 (2015)

DOI: $10.1051 /$ metrology $/ 201501002$

(c) Owned by the authors, published by EDP Sciences, 2015

\title{
Calibration of currentmeters in direction: results obtained on a stock of instruments with a new calibration platform
}

M. Le Menn, L. Pacaud

SHOM, 13 rue du Chatellier, CS 92803, 29228 Brest Cedex

\begin{abstract}
In order to preserve their efficiency, submarine tidal turbines and other marine renewable energy systems need an accurate knowledge of sea currents directions. The speeds and directions of these currents can be measured in a cell of water with currentmeters (AQD) or on a water column with profilers called AQP or ADCP which stands for Acoustic Doppler Current Profiler. When these instruments are installed on carriers, mooring lines or mooring cages, it is necessary to correct the measured data in heading, roll and pitch, in order to interpret them in a terrestrial referential. In this way, they are equipped with a magnetic compass and tilt sensors. Magnetic environment influences compasses responses. There was no system to calibrate AQD and AQP in their using configurations. Following a method perfected in 2007 in order to correct angle errors and warranting a measurement uncertainty in the case of instruments fixed in mooring cages, a calibration platform for magnetic compasses and tilt sensors has been built. In order to show the interest and the corrections allowed by this equipment, this publication shows the effects of the instrumentation of a DORA mooring cage on the compass response of an AQD. The measurements underscore that the initial compass error of $\pm 4{ }^{\circ}$ peak-peak is between $-10^{\circ}$ and $+5.7^{\circ}$ after installation in the cage. This publication shows also the results obtained after the calibration of a stock of 30 AQD currentmeters and 22 AQP profilers. The manufacturer specifications of $\pm 2{ }^{\circ}$ in heading are respected only in $37 \%$ of cases for AQD's and $23 \%$ for AQP's. The heading and roll specifications of $\pm 0.2^{\circ}$ are never respected but $86 \%$ of profilers and $53 \%$ of currentmeters show errors less than $2^{\circ}$.
\end{abstract}

\section{Introduction}

Speed and direction of sea currents were initially measured with rotor currentmeters. Rotor currentmeters have been replaced by acoustic technologies.

The acoustic currentmeters (AQD) measure the Doppler Effect in a water cell by emitting acoustic pulses and receiving echoes. The Doppler profilers (AQP or ADCP as Acoustic Doppler Current Profiler) measure speeds in a user specified number of cells from the instrument out to a maximum range that depends on acoustic scattering conditions.

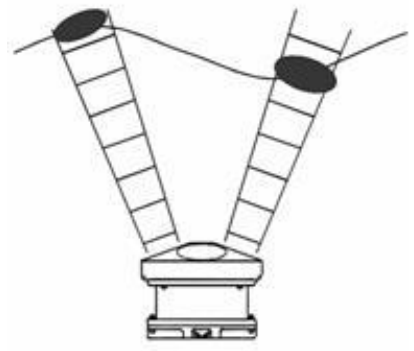

Standard geometry

Figure 1: scheme of an ADCP emitting to the surface. Doc. Teledyne R.D. Instruments

The currentmeters and the profilers measure speeds $\left(V_{l}\right.$, $\left.V_{2}, V_{3}\right)$ in the beams directions. The beams are slanted to $20^{\circ}, 25^{\circ}$ or $30^{\circ}$ ( $\beta$ angle) according to the instrument. $\beta$ being known, speeds $\left(V_{x}, V_{y}, V_{z}\right)$ can be process in the beams frame (1). Moreover, these instruments are equipped with a 'flux-gate' compass to retrieve currents values $(U, V, W)$ to the magnetic North ( $\Omega$ heading angle), and then, with the magnetic declination, in reference to the true North (2). Their horizontality is corrected with 'tilt sensors'. In equation (2) angles $\Psi$ represents the roll and $\theta$ the pitch angle $(C=\cos$ and $S=$ $\sin )$.

$$
\begin{aligned}
& {\left[\begin{array}{l}
V_{x} \\
V_{y} \\
V_{z}
\end{array}\right]=\left[\begin{array}{ccc}
\frac{2}{3 \sin (\beta)} & \frac{-1}{3 \sin (\beta)} & \frac{-1}{3 \sin (\beta)} \\
0 & \frac{-1}{\sqrt{2} \sin (\beta)} & \frac{1}{\sqrt{2} \sin (\beta)} \\
\frac{1}{3 \cos (\beta)} & \frac{1}{3 \cos (\beta)} & \frac{1}{3 \cos (\beta)}
\end{array}\right]\left[\begin{array}{l}
V_{1} \\
V_{2} \\
V_{3}
\end{array}\right] \quad(1)} \\
& {\left[\begin{array}{l}
U \\
V \\
W
\end{array}\right]=\left[\begin{array}{ccc}
C_{\psi} C_{\Omega} & \left(-S_{\psi} S_{\theta} C_{\Omega}+C_{\theta} S_{\Omega}\right) & \left(S_{\psi} C_{\theta} C_{\Omega}+S_{\theta} S_{\Omega}\right) \\
-C_{\psi} C_{\Omega} & \left(S_{\psi} S_{\theta} S_{\Omega}+C_{\theta} S_{\Omega}\right) & \left(-S_{\psi} C_{\theta} S_{\Omega}+S_{\theta} C_{\Omega}\right) \\
-S_{\psi} & -C_{\psi} S_{\theta} & C_{\psi} C_{\theta}
\end{array}\right]\left[\begin{array}{l}
V_{x} \\
V_{y} \\
V_{z}
\end{array}\right]}
\end{aligned}
$$

The currentmeters and profilers data are used to make sea currents charts, 3D models of currents but also to assess rivers flows. With the development of marine renewable energy systems and particularly, submarine tidal turbines, the need appeared of a more accurate knowledge of currents directions. Unlike several kinds of wind turbines, most of submarine tidal turbines are installed in fixed frames.

The Figure 2, from the reference [1], shows the efficiency of a turbine as a function of the rotation speed for

\footnotetext{
${ }^{\text {a }}$ Corresponding author: marc.lemenn@SHOM.fr

This is an Open Access article distributed under the terms of the Creative Commons Attribution License 4.0, which permits unrestricted use, distribution, and reproduction in any medium, provided the original work is properly cited.
} 
different incidence angles from 0 to $30^{\circ}$. Beyond $20^{\circ}$, the turbines efficiency decreases strongly. Thereafter, it is important to determine accurately the direction of sea currents to direct correctly the frame of submarine tidal turbines.

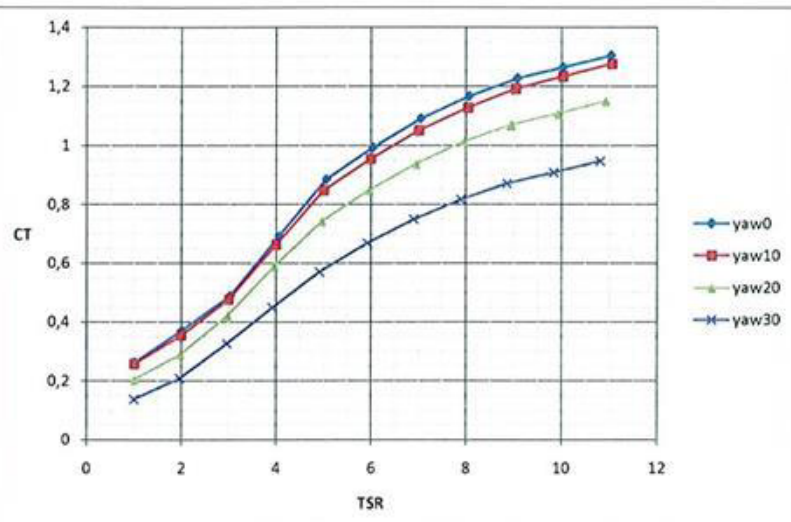

Figure 2: efficiency of a turbine to different incidence angles of the fluid, from K. M. Loland Thesis [1]. CT = efficiency, TSR = rotor speed.

There was no tool to warrant the accuracy of sea currents directions measurements, so that manufacturers specifications. The SHOM has a stock of 43 currentmeters and 47 profilers. Then, a calibration platform was developed to respond to these needs.

\section{Installation of the calibration platform}

The platform has been specified to offer uncertainties on reference angles better than currentmeters specifications which are: $\pm 2^{\circ}$ for the heading and $\pm 0.2^{\circ}$ for tilt angles, with a precision of $0.5^{\circ}$ for the heading and $0.1^{\circ}$ for the tilt sensor.

The tilt sensor is necessary to correct the instrument's slant and to retrieve the true speed and the true distance of measurement cells in the case of profilers. The heading angles are measured by a magnetic compass which is sensible to anomalies of the terrestrial magnetic field. The anomalies can be created by hard and soft iron elements situated in the vicinity of the compass. Then, it is necessary to calibrate them in their using environment which is generally composed of a mooring cage equipped with many other instruments. Consequently, the platform must be able to support loads of about $800 \mathrm{~kg}$.

The platform implantation place was chosen after a magnetic map-making to determine an area where gradients are inferior to $5 \mathrm{nT} \mathrm{m}^{-1}$. The figure 3 shows the magnetic anomalies obtained after correcting for the temporal and secular variations of the terrestrial field.

The conception follows feasibility experiences realized in 2007 [2] and it was the subject of a second publication in 2014 [3]. In the area with the lower gradients, a flat concrete block was constructed with a measured maximum slope of $3 \mathrm{~mm} \mathrm{~m}^{-1}$ or $0.18^{\circ}$ and an average roughness equivalent to an uncertainty of $0.15^{\circ}$. A nonmagnetic table was then constructed, that can be rotated and tilted. The inclination is measured using a digital protractor calibrated in a reference laboratory with an uncertainty of $0.12^{\circ}$. After the setup of this platform, another magnetic mapping was carried out to verify the uniformity of the field.

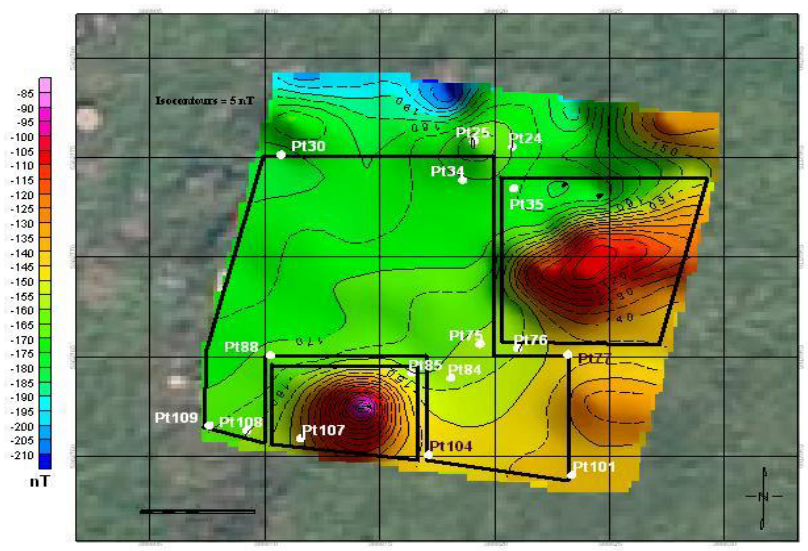

Figure 3: mapping realized with a proton magnetomer GSM 19

The concrete block was graduated with a GPS Leica receiver used in RTK (Real Time Kinematic) mode (differential measurements from a ground station).

The expanded uncertainty on the reference directions was assessed to be $0.55^{\circ}$ (according to [4]). Furthermore, the reproducibility of measurements $\left(0.19^{\circ}\right)$, so that the homogeneity of the platform's field were assessed. Finally, the expanded uncertainty on reference directions, taking into account the positioning uncertainties and the magnetic uncertainties is about $0.97^{\circ}$. If the precision (from manufacturer's specifications) of instruments under calibration is added, it leads to an expanded uncertainty of $1.1^{\circ}$

\section{Example of results: effects of an instrumented DORA cage}

A Nortek AQD n 2060 currentmeter was calibrated on the platform and then, installed in a DORA cage equipped with its ballast, a releaser RT861 iXsea, a turbidimeter WETLAB, a SBE 39 and a flash lamp.

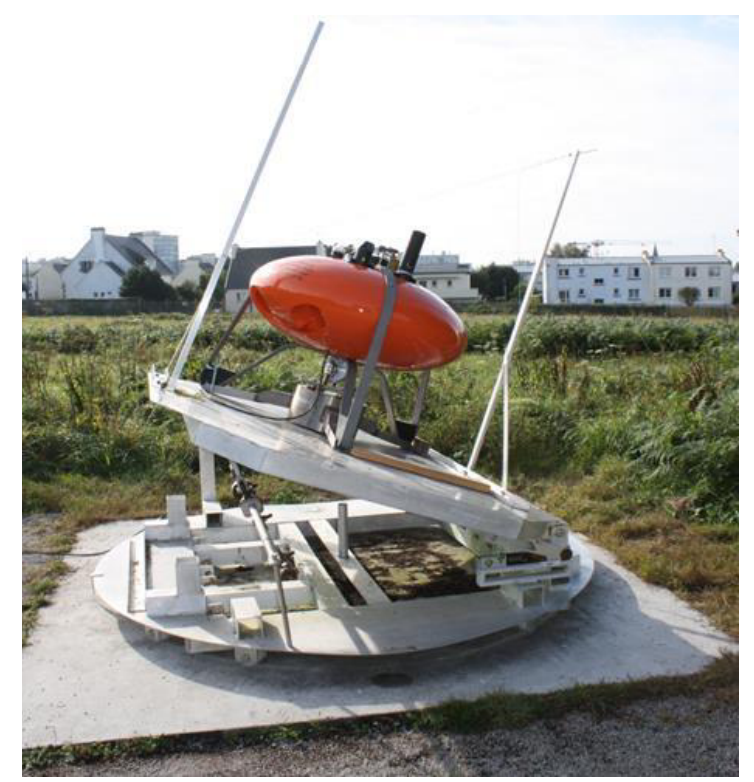

Figure 4: DORA cage (orange) and its frame and instruments put on the slanted platform for the tilt calibration. 


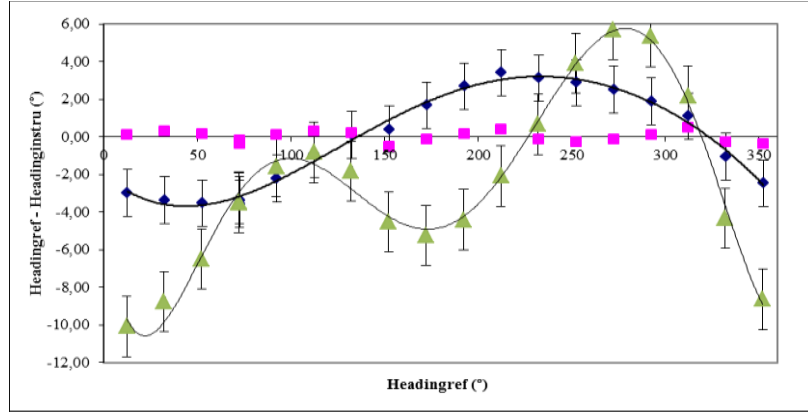

Figure 5: blue squares, error function of the ADQ $n^{\circ} 2060$ alone. Green triangles, error function after installation in an instrumented Dora Cage. Mauves squares, residuals of the correction.

Before installation in the DORA cage, the amplitude of the compass error function was less than $\pm 4^{\circ}$. After installation in the cage, the error function shows deviations between $-10^{\circ}$ and $+5.7^{\circ}$ and 2 maximums instead of one. Errors can be considered and corrected by a polynomial or with the so-called 'd'Archibald Smith' relation [5]:

$\Gamma=A+B \sin (\Omega)+C \cos (\Omega)+D \cos (2 \Omega)+E \sin (2 \Omega)$

It gives corrected deviations (Figure 5) with a standard deviation of $0.59^{\circ}$. The responses of pitch and roll sensors remain unchanged. They can be corrected with polynomial relations. These relations are programmed in the software Hypack [6] used to acquire and treat current data.

\section{Statistics about SHOM AQD and AQP stock}

30 NORTEK currentmeters AQD 200, 2000 and $6000 \mathrm{~m}$ of the SHOM stock were calibrated on the platform, so that 22 NORTEK AQP $400 \mathrm{kHz}, 600 \mathrm{kHz}$ and $1 \mathrm{MHz}, 5$ NORTEK AWAC and 3 Teledyne RDI ADCP Workhorse.

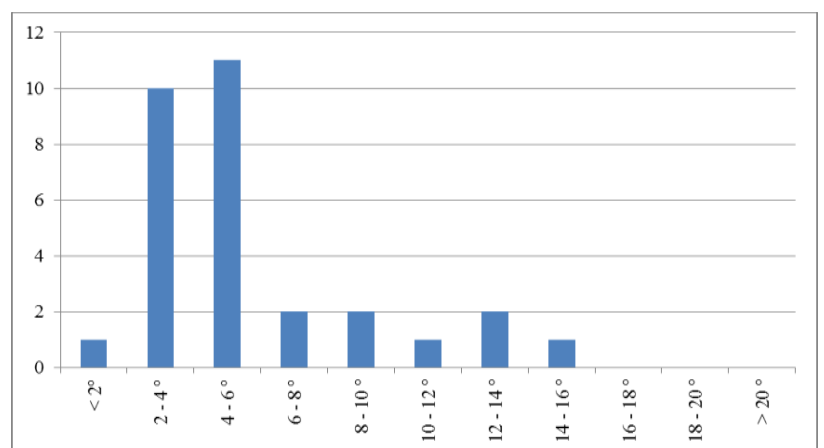

Figure 6: statistics about SHOM's AQD 200, 2000 and 6000 $\mathrm{m}$, heading calibration (errors peak-peak).

Concerning the maximum peak to peak heading deviation of the 30 AQD: $87 \%$ of them have deviations inferior to $10^{\circ} \mathrm{pp}$ and $37 \%$ are in the specification $\pm 2{ }^{\circ}$ given by the manufacturer. $10^{\circ}$ is the specification required to make sea current charts. $13 \%$ present deviations between $10^{\circ} \mathrm{pp}$ and $20^{\circ} \mathrm{pp}$. As regard to the pitch and roll errors, no-one is in the specification $\pm 0.2^{\circ}$ given by the manufacturer (and hard to check in practice): $53 \%$ in pitch and $70 \%$ in roll present errors inferior to $2^{\circ} \mathrm{pp}$. For the others, errors are between 2 and $4^{\circ}$.

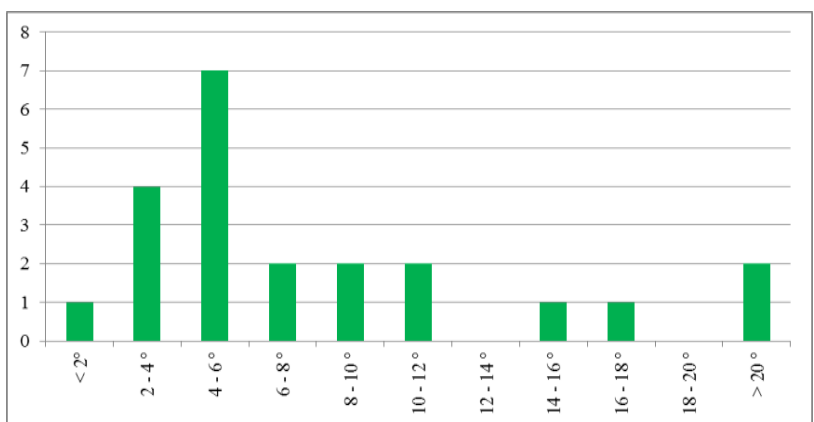

Figure 7: statistics about SHOM's AQP $400 \mathrm{kHz}, 600 \mathrm{kHz}$ and $1 \mathrm{MHz}$, heading calibration (errors peak-peak).

Concerning the heading error peak to peak of the 22 AQP: $73 \%$ of instruments have deviations inferior to 10 ${ }^{\circ}$ pp and only $23 \%$ are in the $\pm 2{ }^{\circ} .18 \%$ of instruments have deviations between $10^{\circ}$ and $20^{\circ}$ but two of them present very high errors: $24.0^{\circ} \mathrm{pp}$ and $34.4^{\circ} \mathrm{pp}$. As regard to the pitch and roll calibration, no-one is in the specification $\pm 0.2^{\circ}$ given by the manufacturer but $86 \%$ present errors inferior to $2^{\circ} \mathrm{pp}$ in pitch and roll, which is a good and important result for profilers. The others have errors between 2 and $4^{\circ}$.

Concerning the peak to peak heading deviation of the five AWAC and the three Workhorse: $100 \%$ of AWAC have errors less than $10^{\circ} \mathrm{pp}$ but among them, four are in the part $6-8^{\circ}$. One Workhorse presents errors inferior to \pm 2 ${ }^{\circ}\left(2.8^{\circ} \mathrm{pp}\right)$, one is in the part $4-6^{\circ}$ but another has an error of $21.5^{\circ} \mathrm{pp}$. As regard to the pitch and roll calibration, one time again, no-one is in the specification $\pm 0.2^{\circ}$ given by the manufacturer. The AWAC present errors distributed between 0.5 and $5^{\circ} \mathrm{pp}$. The Workhorse profilers present errors between $1^{\circ}$ and $3^{\circ}$.

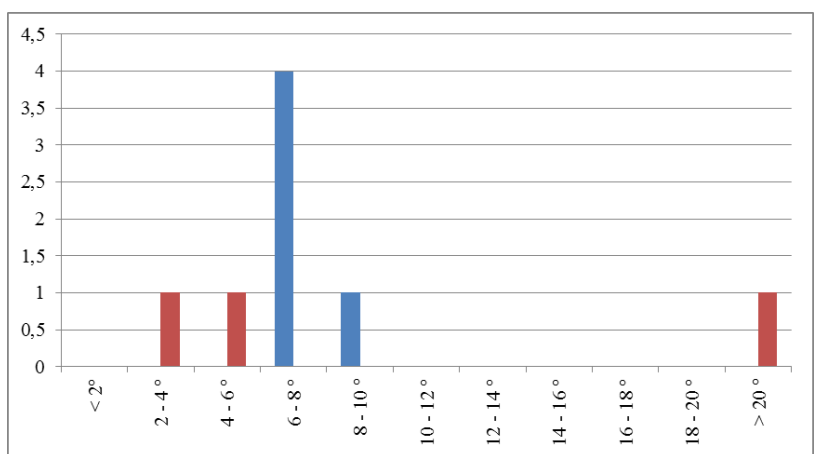

Figure 8: statistics about five AWAC (in blue) and three Workhorse (in red), heading calibration (errors peak-peak).

\section{Conclusions}

The metrological specifications of the calibration platform for compasses and tilt sensors allow:

- to take into account and to correct errors on direction measurements brought by the instruments installed on mooring cages;

- to check the specifications given by the manufacturers of currentmeters and profilers. 
Most of them don't enter in the tolerances of these specifications and some of them, from their acceptance, present inacceptable errors, which can be demonstrated and corrected. A calibration of heading and tilt sensors is then essential before using.

After having made the calibration of this stock of instruments, it stays to determine how the compasses and tilt sensors responses can evolve during the time, in order to be able to adjust their calibration periodicities.

As the direction calibrations are now mastered, we have to work on the set up of a method to control the accuracy of the Doppler effect measurements.

\section{References}

1. Kari Medby Loland, 'Wind Turbine in Yawed Operation', Master of Science in Energy and Environment, Norwegian University of Science and Technology, June 2011.

2. M. Le Menn, M. Le Goff, 'A method for absolute calibration of compasses', Measurement Science and Technology, 18, 1614-1621, (2007).

3. M. Le Menn, A. Lusven, E. Bongiovanni, P. Le Dû, D. Rouxel, S. Lucas, L. Pacaud, 'Current profilers and currentmeters compass and tilt sensors errors and calibration', Measurement Science and Technology, 25 (2014) 085801 (6pp).

4. BIPM, JCGM 100:2008, GUM 1995 with minor corrections, 'Evaluation of measurement data - Guide to the expression of uncertainty in measurement (GUM)', First edition, Sept. 2008.

5. W. Denne, 'Magnetic compass deviation and correction', $3^{\text {rd }}$ edition Brown, Son and Fergusson, 165 pp, 1998.

6. HYPACK, Hydrographic survey software, HYPACK 56 Bradley Street, Middletown, CT06457, USA. 